\title{
Redefining Workplace Wellness: Wearable Technology and Corporate Wellness
}

\author{
Priyadharshini S K*
}

\begin{abstract}
The recent advancements in wearable technology have changed the way we look at health and wellness. This new age development has made its way into the world of corporate and given the benefits, it is meant to stay there for some time. These technologies have been increasingly incorporated in the corporate wellness initiatives with a focus on improving the overall health and wellness of the employees. There are many advantages to a healthy workforce including increased productivity and lower health insurance premiums. A growing trend in corporate wellness programs is to offer wearable fitness trackers to employees to help them monitor their activities so that they can make better choices about their health and lifestyle. This article explores the current trend in the corporate wellness initiatives and also discusses the benefits, barriers and threats of this new age development.
\end{abstract}

Keywords: Wearable Technology, Corporate Wellness Programs, Data Privacy, Fitness Ecosystem

\section{Introduction}

The modern workplace and culture have undergone a sweeping transformation in the last few years. One area that sees continued growth and change revolve around the corporate wellness space. Mental and physical components have gained prominence over the

\footnotetext{
*URUFIT, Chennai, India; priya.lifecool@gmail.com
} 
usual components. The new age corporate wellness programs are becoming more holistic, treating an employee's mind and body. With fitness trackers becoming more popular among "health conscious" individuals, companies have shifted their attention to wearable technologies at the workplace. In this article, the terms fitness/activity trackers, wearables and wearable technology/ devices are used interchangeably to represent the wearable technology that are in vogue. The idea of a device tracking one's steps, heart rate, and various other biomarkers isn't new per se. What's new is the level of sophistication and the steady growth of them inside the workplace. According to a PwC report (2016), by 2020 more than 75 million wearables will infiltrate the workplace.

Wearable technology refers to accessories and clothing incorporating computer and advanced electronic technologies. Examples of wearable technology devices include fitness trackers/ bands, smart glasses, smart watches, smart clothing, and other wearable devices. With technology continually getting better, companies can use this golden opportunity to utilise this tool to further develop and enrich their wellness initiatives. There is no denying the popularity of wearable technology. In India alone, the wearable market is bound to see a dramatic increase in usage from 56 million users in 2017 to 65 million users in 2023 (Statista, 2019), which clearly means that a wearable technology is more than just a fashion statement for the users. This paper attempts to convey to the readers, the current trend of using wearable technology as part of corporate wellness in India and globally, their advantages and their drawbacks.

\section{Role of Wearable Technology in the Workplace}

The availability and relevance of health-related technology are rapidly evolving. These technologies offer unlimited potential to minimise and/or mitigate barriers associated with achieving optimal health, performance, and readiness (Teyhen et al, 2014). Wearables like FitBit, Apple watch, MI band, etc. have gained traction. With a focus on fitness trackers, there are more and more new entrants into this space. As any market goes, only a few survive to see the growth. But the key here is, the race had begun, signalling the wearable movement we see today where adoption 
has more than doubled in the last couple of years. According to a report by Deloitte (2018), "Wearable technologies such as smart watches, smart glasses, hearables, and exoskeletons can help company leaders navigate by augmenting employees' physical and perceptual capabilities, amplifying their physical strength, lucidly conveying detailed task instructions as needed, facilitating virtual interactions, and alerting for hazards. All of this has the potential to significantly boost productivity and safety".

If anything, technology has made workers more valuable by enhancing their physical and perceptual abilities. This new generation of wearable technology is giving the workforce more strength, endurance, vision, hearing, and awareness. They are enhancing their effectiveness and productivity and are helping in keeping people safe. They also help them overcome physical limitations and give an opportunity to the management to make new ways to plan for the future workforce.

\section{The New Age Workplace and New Technologies}

The impact of technology on work has seen both sides. While some have eliminated jobs and displaced people; others have made employees more productive. The primary objective of any wearable technology is to make employees more productive and more capable in the workplace. The increased use clearly states that wearables have a significant impact on the workforce and the companies that employ them (Meerkerk, 2017). All of this comes at a time when there are rapid technological changes happening in the workplace. How exactly are these technologies augmenting the employer and the organisation as a whole? A study by The Workforce Institute (2014) states that there is a heightened belief that wearable technology will take off in the workplaces. The report also argues that these intelligent devices provide organisations with uncharted data collection points to greatly improve safety, productivity, collaboration, and overall workplace effectiveness.

Employers are increasingly seeing the value that wearable technology can provide to employees and to the organisation. A report by Endeavors Partners (2018) states that employers in the United States have plans to integrate more than 13 million fitness 
tracking devices into corporate wellness programs. This rightly points out that to the fact that, the technology allows employees to individually track their fitness progress and adds an element of gamification and social support-all of which can greatly influence the results.

\section{Employee's Perception and Attitude towards Wearable Technology at the Workplace}

While it is understood that the wearable technology has the potential to unlock a new world of opportunity for both employers and employees by offering key information to understand and manage the employees and increase their engagement, how well is it received by the employees? The study by The Workforce Institute (2014) points out that countries like India, China and Mexico were ahead and optimistic when it comes to workplace benefit from wearables when compared to the US. In the US only $48 \%$ of the study respondents saw benefits from a wearable in the workplace, whereas in $96 \%$ of the Mexicans, $94 \%$ of Chinese and $91 \%$ of Indians felt that they see a greater benefit from wearables at the workplace. This could be attributed to the age bracket of the working population and their general adaptation towards new and emerging technology. PwC's report in 2016 , noted that $65 \%$ of the employees using wearable technology services offered by the employers think that technology has a real role to play in their health and wellbeing. The feedback on exercise and diet based on their activities have been helpful to them. The report also points out that $61 \%$ of employees felt that the active role by the employer in their health and wellbeing was indeed welcome.

\section{Benefits of Using a Wearable Technology at Workplace- Company/Employer Perspective}

A company whose employees use wearable devices experience a positive return on investment. Also, these benefits extend beyond revenue. Wearable-based wellness programs increase productivity, employee loyalty and time spent on the job. Effective wellness programs can reduce absenteeism while simultaneously promoting productivity. On the other hand, unhealthy employees are more 
likely to disengage and are $43 \%$ more likely to have increased stress and anxiety (Draper, 2018). Employers arguably have the power to influence employees' behaviours. Introducing wearable technology to the workplace would provide employers with clearer pictures of employee health, enabling them to develop more targeted initiatives and communications to encourage healthier activity levels. Corporates are increasingly incorporating technology into their wellness initiatives with the aim to improve overall employee health, with special focus on physical and mental health. Some of these initiatives are being developed in partnership with health insurers to provide access to activity and wellness devices (BBC, 2018), along with program designs that are cost-effective to manage for employees. Eventually, this method will lead to a more productive workforce and it also appeals to serve as an employer branding initiative for the prospective employees.

There are a number of benefits for employers who promote wearable technology and create a "fitness ecosystem" in the workplace. Some of the top benefits are:

\subsection{A Workplace Culture Focused on Health and Well-Being}

For years, work environments have made productivity and profits the top priorities, while health and wellness received little attention. While the profits and production metrics are critical to the company's survival, the wellbeing of their workforce is directly correlated to the overall big picture. One way to improve the workplace culture is to get and sustain employees to operate on the same page. Achieving productivity is one thing, but getting the employees to feel better as a collective whole is even more important. With the entry of fitness and activity trackers, along with other wellness devices tied to specific outcomes have moved health and wellness to the forefront of workplace culture. This, in turn, creates a stronger sense of camaraderie, happiness and improves the overall employee retention.

\subsection{Improved Lifestyle Disorder Management}

According to the Centers for Disease Control and Prevention, one in three Americans has Prediabetes. Of those, 90\% do not realise they have it. Another 30 million adults in America are living with 
Diabetes. It is the seventh leading cause of death in America. High blood pressure is another chronic disease that requires constant monitoring and management. India is no different when it comes to Lifestyle Disorders. According to a WHO (2015) report, India has 70 million people living with Diabetes and which is expected to grow to 98 million in the next 5 years. This alarming increase can be attributed to an unhealthy lifestyle. Although heredity plays an important role in the development of Type 2 Diabetes, it can take a back seat with behavioural and lifestyle modifications. Active physical life and mindful eating can help prevent and control these Lifestyle Disorders. With fitness trackers and other wearable devices, people can monitor their physical activity, what they are eating on a daily basis and more. This can lead to improved overall health and, ultimately, increased productivity due to a decreased rate of workplace absences related to illnesses and other ailments.

\subsection{Incentivise Healthy Habits}

Although a company might be ready for the new age corporate wellness challenge, involvement and engagement from the employees is key to a successful wellness program. How exactly to get users engaged? Healthy Behavior Incentive Programs have been widely used to encourage and sustain lifestyle modifications. There have also been studies which claim that financial incentives can effectively reduce unhealthy habits and increase physical activity among individuals (Liao, 2018). These ideas have been extensively adopted by new age health and wellness technology.

The rising use of smartphone apps with built-in incentives, such as points or badges, has turned health monitoring into a game. FitBit for example gives out badges and appreciation to people achieving the targeted milestone. The individual can compare and compete with friends and others in the community. These wearables emphasise accountability, while also encouraging movement toward a common goal, creates competition and, in the end, more engaged employees. In fact, companies have taken the path of gamification and expanded it further by putting programs in place that incentivise healthy employee habits. Companies like PwC are known for their healthy behavior incentive programs. 


\subsection{Health Care Cost Savings}

While focusing on health and wellness in the workplace, employers' sense that it reduces their health associated costs due to fewer illnesses. Bringing more focus on wellness also helps employees in reducing the chances of experiencing a major illness (or even chronic illness). Coupling wearable technology with employer-sponsored workplace health and wellness programs can help to offset these rising costs. For example, a more physically active workforce may not need to see a doctor as frequently. This would mean that the employee and, possibly, his/her immediate dependents may have a reduced need for a robust and often, costlier health insurance plan. That translates to possible significant savings to both the employee and employer. This technologyenabled corporate wellbeing initiatives are a means to tackle absenteeism costs for employers.

\subsection{The Flip-Side to Tech Enabled Corporate Wellness}

With fitness trackers in the workplace, bosses can monitor employee's every step maybe even more. This constant health surveillance is a double-edged sword. It should also be noted that some employees find these real-time tracking of their daily movements via the company provided digital tracker intrusive. The fueling boom of these digital trackers only suggests that the data acquired are an increasingly valuable source of workforce health intelligence for employers and insurance companies. The sheer volume of highly sensitive health data gathered from individual employees is raising privacy concerns and adding a new dimension to the relationship between employees and their employers. Employees are giving up more insights about themselves to the third party more than they realise. These sophisticated wearable devices are measuring more than just the steps and distance walked but also the hours an employee spends in a sedentary state, continuous heart rate, and sleep duration and quality. Is it that the employees are voluntarily signing up for these digital health monitoring or in the name of corporate-wellness are they lured by cash, reduced premiums, or reimbursements for co-payments and deductibles? It is too early to answer such questions. One has to wait and watch how the tide sways. Since the user of the device 
(employee) is voluntarily giving up data, companies enjoy greater legal flexibility in how it is used.

The privacy and workforce specialists warn that the data could be used against the individual to favour the healthiest employees while punishing or stigmatising those who are less healthy, or who show signs of unhealthy behaviour such as heavy drinking or drug use (Rowland, 2019). The data recorded from the wearable devices allows the employer to track significant data on employees, including information such as location, hours worked, rest breaks and even activity levels. The more employers know about their employees' lives, it is quite possible that these data will have effects on whether an employee will be retained, promoted, demoted, or even laid-off.

\subsection{Wearables at Work in India}

It is safe to say that India is leading the race in embracing wearable technology at work (The Workforce Institute, 2014). People in India have particularly responded very positively towards the incorporation of wearable devices in their workplace (91\%). Aligning with this study, results from Accenture Digital Consumer Tech Survey (2014), found that more than 52\% of the respondents are interested in buying wearable technologies to track their physical activities and to manage their personal health. Among the six countries studied, Indian consumers were found to be most interested in buying wearable technologies like Fitness Monitors, $(80 \%)$, smart watches $(76 \%)$ and Internet-enabled eyeglasses $(74 \%)$. Going by the numbers (Statista, 2019), the wearable technology users are expected to grow up to 65 million users by 2023, most of them fall between the age group of 25-34 years (48\%) followed by people aged 18-24 (39\%). The increased use among the working population (25-34 years) is reflected in the higher acceptance and positive outlook towards the benefits of wearable technology at the workplace.

\subsection{Digitally Enhanced Corporate Wellness - Key to a Healthier, Happier Workplace}

When an organisation decides to change the culture and make the workforce healthier, it is important to understand if the 
organisation is ready for such a change. And, if so, what are they ready to change? Behavior changes cannot happen overnight and hence, understanding the readiness to change and the stage in which they are can help in successful acceptance and implementation. If the organisation's objective is to create a healthier workplace, then it is best to start from the top. When managers and leaders foster a culture of wellness and characterise it, employees notice and are more likely to follow suit. Another important factor for successful acceptance of the digitally enhanced workplace is understanding what motivates (intrinsic and extrinsic) the individual to participate and getting the incentives right. In order to have a successful and healthy workplace, it is also necessary for an organisation to identify solutions that boost health, wellness and productivity of people across the entire workplace. With a thorough understanding of the needs and with the careful and sensitive implementation of the digitally enhanced corporate wellness, the company can reap the positive impact on productivity, health and morale of the employees. Taking health to employees through wellness initiatives leads to a more engaged, loyal and healthy workforce. The benefits of these well-informed and well-accepted wellness initiatives are endless. But without the right approach, they maybe a recipe for disaster.

\section{Conclusion}

When employers commit to workplace wellness initiatives, putting health and wellness of employees first, they create a workplace culture of care, productivity and engagement. One must acknowledge that the workplace wellness wearable technology programs can provide employers with enormous data about their employees and the workplace which can be put to best use. Employees are usually willing to use technology to better themselves, their lifestyle and their health. Implementing wearable technology into the workplace not only can create a positive environment for the entire workplace, but it can help improve safety, lower healthcare costs, and monitor health and wellness. On the other hand, given the large number of data collected, employers must address the concerns the employees have about fitness tracking and privacy. Generally, employees worry if it could lead 
to discriminating them and impact their boss's perception of them because of their lifestyle choices. To understand if wearable technology based wellness initiative is worth the effort, it is important to understand the underlying motive behind the implementation. But for most organisations, the benefits of providing fitness devices far outweigh the costs. Apart from the increase in productivity, employers also report many indirect benefits, such as increased morale and reduced absenteeism. The social element of a wearable based wellness initiative helps people to stay motivated and creates a culture of support for all employees. This increases engagement and helps employee become more productive. When employers invest in the health, it is a winwin. Technology will continue to play an important role in overall employee health for years to come. Workplace-based wellness programs coupled with personal technology can lead to significant cost savings for both employers and employees while also helping to create a more engaged and healthier workforce.

\section{References}

Accenture. (2014). Racing Toward a Complete Digital Lifestyle: Digital Consumers Crave More. Retrieved April 30, 2019, from https:// www.accenture.com/t20150826T034315_w_/pl-en/__ acnmedia/ Accenture/Conversion-Assets/ Careers/ Documents/ LandingPage/ 1/ Accenture-Digital-Consumer-Tech-Survey-2014.pdf

BBC. (2018, September 20). John Hancock adds fitness tracking to all policies. Retrieved May 1, 2019, from https:// www.bbc.com/ news/ technology-45590293

CDC. (2019). Prediabetes - Your Chance to Prevent Type 2 Diabetes | CDC. (n.d.). Retrieved April 30, 2019, from https:// www.cdc.gov/ diabetes/ basics/prediabetes.html

Draper, S. (2018, July 13). How to Utilize Wearable Tech for a Successful Workplace Wellness Program. Retrieved May 1, 2019, from https://www.wearable-technologies.com/2018/07/how-to-utilizewearable-tech-for-a-successful-workplace-wellness-program/

India Today. (2018, November 23). Diabetes epidemic: 98 million people in India may have type 2 diabetes by 2030. Retrieved May 1, 2019, from https://www.indiatoday.in/education-today/gk-currentaffairs/ story/98-million-indians-diabetes-2030-prevention-13941582018-11-22

Liao, J. M., \& Navathe, A. S. (2018). Incentivizing healthy behaviors at scale: closing the gap between science and population-level 
implementation. JAMA Network Open, 1(8). doi:10.1001/ jamanetworkopen.2018.6173

Marketwired. (2014, August 15). Endeavour Partners' Consumer Behavior Study Points to Uncertain Future of Wearable Devices. Retrieved from http:/ / www.marketwired.com/press-release/endeavour-partnersconsumer-behavior-study-points-uncertain-future-wearable-devices1938954.htm

Meerkerkonline. (2017). Wearable Technology Can Increase Productivity, Study Finds. (2017, September 30). Retrieved May 1, 2019, from https:// meerkerkonline.com/wearable-technology-can-increaseproductivity-study-finds /

PricewaterhouseCoopers. (2016). The wearable life 2.0: connected living in a wearable world. Retrieved April 28, 2019, from https:// www.pwc.com/us/en/services/consulting/library/consumerintelligence -series/wearables.html

Rowland, C. (2019, February 16). With fitness trackers in the workplace, bosses can monitor your every step - and possibly more. Retrieved April 30, 2019, from https:// www.washingtonpost.com/ business/ economy/with-fitness-trackers-in-the-workplace-bosses-can-monitoryour-every-step--and-possibly-more/2019/02/15/75ee0848-2a4511e9-b011-d8500644dc98_story.html

Schatsky, D., \& Kumar, N. (2018, July 25). Workforce superpowers wearables are augmenting employees' abilities. Retrieved April 30, 2019, from https://www2.deloitte.com/ content/dam/ insights/ us/ articles/4599_Superhuman-workforce/DI_Workforcesuperpowers.pdf

Statista. (2019). Wearables - India | Statista Market Forecast. (2019). Retrieved April 30, 2019, from https://www.statista.com/ outlook/319/119/ wearables/ india

The Business Wire. (2014, October 27). Kronos Survey Indicates Workers around the World are Poised to Embrace Wearable Technology. Retrieved May 1, 2019, from https:// www.businesswire.com/ news /home/ 20141027005524/en/Kronos-Survey-Workers-World-PoisedEmbrace-Wearable

Teyhen, D. S., Aldag, M., Centola, D., Edinborough, E., Ghannadian, J. D., Haught, A., \& Parramore, D. J. (2014). Incentives to create and sustain healthy behaviors: technology solutions and research needs. Military Medicine, 179(12), 1419-1431. 